\title{
Possible emittance growth induced by nonlinear space charge fields for arbitrary particle distributions
}

\author{
Takashi Kikuchi (菊池 崇志) \\ Department of Nuclear System Safety Engineering, Nagaoka University of Technology, \\ 1603-1 Kamitomioka-machi, Nagaoka, Niigata 940-2188, Japan \\ Kazuhiko Horioka (堀岡 一彦) \\ Department of Energy Sciences, Tokyo Institute of Technology, \\ 4259 Nagatsuta-cho, Yokohama, Kanagawa 226-8502, Japan \\ (Received 14 April 2016; published 16 June 2016)
}

\begin{abstract}
A procedure to obtain a ratio of beam radii at final and initial states in arbitrary particle distributions is proposed, and is applied to the estimation of possible emittance growth for Gaussian and thermal equilibrium distributions. The ratios are estimated for Gaussian and thermal equilibrium distributions as a function of tune depression. The possible emittance growth as a function of tune depression and nonlinear field energy factor is also estimated with and without a constant radius ratio approximation. It is confirmed that the possible emittance growths are almost the same in comparison to the cases with and without the constant radius ratio approximation at each distribution.
\end{abstract}

DOI: 10.1103/PhysRevAccelBeams.19.064201

\section{INTRODUCTION}

Transport of intense charged particle beams with lower temperature is a key issue in applications for high-current beam such as inertial confinement fusion, high energy density science, and warm dense matter research, driven by heavy ion beams [1-5]. The emittance, which is a useful index to express a beam quality, is defined as a statistical value in phase space [6-8]. The charged particle beams should be transported with as little emittance growth as possible, because a large emittance beam is focused into a large spot size on a target.

In an azimuthally symmetric beam, the nonlinear evolution of the unnormalized emittance $\varepsilon$ along transport distance $s$ is written by [6]

$$
\frac{\mathrm{d} \varepsilon^{2}}{\mathrm{~d} s}=r_{\mathrm{b}}^{2}\left(-\frac{K}{2 r_{\mathrm{b}}} \frac{\mathrm{d} r_{\mathrm{b}}}{\mathrm{d} s}-\frac{\mathrm{d} E_{\mathrm{sf}}}{\mathrm{d} s}\right)
$$

where $r_{\mathrm{b}}$ is the beam radius, $E_{\mathrm{sf}}$ is the self-field energy, and $K$ is the self-field perveance

$$
K=\frac{2}{\beta_{\mathrm{b}}^{3} \gamma_{\mathrm{b}}^{3}} \frac{I_{\mathrm{b}}}{I_{0}},
$$

\footnotetext{
"tkikuchi@vos.nagaokaut.ac.jp

Published by the American Physical Society under the terms of the Creative Commons Attribution 3.0 License. Further distribution of this work must maintain attribution to the author(s) and the published article's title, journal citation, and DOI.
}

respectively. Here $\gamma_{\mathrm{b}}$ is the relativistic factor, $\beta_{\mathrm{b}}$ is the beam velocity divided by the light speed $c, I_{\mathrm{b}}$ is the beam current, and $I_{0}$ is the characteristic current

$$
I_{0}=\frac{4 \pi \epsilon_{0} m c^{3}}{q}
$$

where $q$ is the charge of beam particle, $m$ is the rest mass of beam particle, and $\epsilon_{0}$ is the permittivity of free space, respectively. Equation (1) indicates that the emittance change is driven by the beam radius and/or the self-field energy changes, when the particle energy and current are being held constant, i.e., the perveance $K$ is held constant.

Reiser developed the expressions for the possible emittance growth due to the nonuniform charge distribution, mismatched beam, and off-centered beam, to introduce the free-energy parameter [9]. Recently, in the case of initially mismatched beam, Osaki and Okamoto generalized the theory to treat an intense bunched beam focused by linear external forces in three dimensions [10]. Struckmeier et al. treated the possible emittance growth between the nonuniform and the uniform (stationary) initial beams [11]. Some beam conditions, such as the Kapchinskij-Vladimirskij $(\mathrm{KV})$, waterbag, parabolic, conical, Gaussian, semiGaussian, and thermal equilibrium distributions, were considered and proposed to analyze the behavior including instability of the beam $[7,8,11]$. The minimum field energy of a charge distribution applies to the uniform charge density in real space associated with the KV distribution in phase space. For this reason, beams with a nonuniform charge distribution (like as a non-KV distribution) are not stationary, and will change toward a more homogeneous charge distribution in real space. 
Beams with a nonuniform charge distribution have an additional field energy in comparison to the beam with the uniform charge density in real space. The additional field energy will be converted into particle kinetic and potential energies. The rms emittance $\varepsilon_{\text {rms }}$ of a general particle distribution $f\left(x, x^{\prime}\right)$ in $x$-direction is defined by [8]

$$
\varepsilon_{\mathrm{rms}}^{2}=\left\langle x^{2}\right\rangle\left\langle x^{\prime 2}\right\rangle-\left\langle x x^{\prime}\right\rangle^{2}=\left\langle x^{2}\right\rangle \frac{\left\langle v_{x}^{2}\right\rangle}{v_{0}^{2}}-\frac{\left\langle x v_{x}\right\rangle^{2}}{v_{0}^{2}},
$$

where $x$ is the each particle position in real space, the prime (') means the derivative $\mathrm{d} / \mathrm{d} s, v_{x}=\mathrm{d} x / \mathrm{d} t$ is the each particle velocity, $v_{0}=\beta_{\mathrm{b}} c$ is the beam velocity, respectively. The value $\langle X\rangle$ indicated by brackets means the average of $X$. When the additional field energy is converted into a particle kinetic energy, hence the beam emittance as indicated as Eq. (4) will be increased as the distribution tends to become more homogeneous. The "possible" emittance growth induced due to the space charge potential via free-energy was introduced $[9,11,12]$. However, the theoretical analysis has been treated with an assumption that the beam radius remains constant for the case of nonuniform charge distribution. While, it is expected that the beam radius increases actively in the case of longitudinal bunch compression, due to the space charge effect [13-16].

In this paper, we develop the calculation procedure for a changing ratio of the beam radii at the final and initial states in arbitrary particle distributions, and hence possible emittance growth is estimated for Gaussian and thermal equilibrium distributions.

\section{POSSIBLE EMITTANCE GROWTH DESCRIBED BY FREE-ENERGY PARAMETER WITHOUT APPROXIMATION OF CONSTANT RADIUS RATIO}

Possible emittance growth $\varepsilon_{\mathrm{f}} / \varepsilon_{\mathrm{i}}$ is written by $[7,8]$

$$
\frac{\varepsilon_{\mathrm{f}}}{\varepsilon_{\mathrm{i}}}=\frac{a_{\mathrm{f}}}{a_{\mathrm{i}}}\left[1+\frac{1}{\sigma^{2} / \sigma_{0}^{2}}\left\{\left(\frac{a_{\mathrm{f}}}{a_{\mathrm{i}}}\right)^{2}-1\right\}\right]^{1 / 2},
$$

where $a_{\mathrm{f}} / a_{\mathrm{i}}$ is the final and initial beam radii ratio $\left(a_{\mathrm{f}}\right.$ and $a_{\mathrm{i}}$ are the final and initial beam radii) and $\sigma / \sigma_{0}$ is the tune depression for a periodic focusing channel. The tune depression, which is an index of space charge strength, is given by the depressed and undepressed phase advances $\left(\sigma\right.$ and $\left.\sigma_{0}\right)$ per lattice period. The undepressed phase advance per lattice period is $\sigma_{0}=k_{\beta 0} L_{\mathrm{p}}$, where $k_{\beta 0}$ is the wave number of betatron oscillation without the space charge effect and $L_{\mathrm{p}}$ is the length per lattice period, respectively. The depressed phase advance (phase advance with space charge) per lattice period is expressed as

$$
\sigma=k_{\beta} L_{\mathrm{p}}=\int_{s}^{s+L_{\mathrm{p}}} \frac{\varepsilon}{r_{\mathrm{b}}^{2}} \mathrm{~d} s
$$

where $k_{\beta}$ is the wave number of betatron oscillation with the space charge effect. The parameters of the periodic focusing channel (strength and lattice period) are being held constant, i.e., the tune depression $\sigma / \sigma_{0}$ is being held constant. Since the beam is assumed to have a final specific value for the radius, we assume that they are implicitly considering a uniform continuous focusing channel. Since the initial beam also has a well-defined radius, we assume that the initial beam is not mismatched $\left(\mathrm{d} r_{\mathrm{b}} / \mathrm{d} s=0\right)$, and the beam is initially in an equilibrium.

From Eq. (7) in Ref. [9], the following relation is described

$$
\left(\frac{a_{\mathrm{f}}}{a_{\mathrm{i}}}\right)^{2}-1-\chi \ln \frac{a_{\mathrm{f}}}{a_{\mathrm{i}}}=h
$$

where

$$
\chi=1-\frac{\sigma^{2}}{\sigma_{0}^{2}}
$$

Here, the free-energy parameter $h$ (and $h_{\mathrm{s}}$ is the free-energy parameter due to space charge) is written as (see Eq. (13) in Ref. [9]), for a nonuniform charge distribution,

$$
h=h_{\mathrm{s}}=\frac{1}{4}\left(1-\frac{\sigma^{2}}{\sigma_{0}^{2}}\right) \frac{U}{w_{0}},
$$

where $U / w_{0}$ is the nonlinear field energy factor. Here $U=w-w_{0}$, and

$$
w=\pi \epsilon_{0} \int_{0}^{r_{\mathrm{p}}} E(r)^{2} r d r
$$

is the field energy per unit length, where $r_{\mathrm{p}}$ is the pipe (vacuum chamber) inner radius, and $E(r)$ is the self-field in the radial direction $r$. The field energy per unit length within the actual beam volume is $w_{0}=\lambda^{2} / 16 \pi \epsilon_{0}$, where $\lambda$ is the line charge density. The nonlinear field energy factor depends on the particle distribution, e.g., $U / w_{0}=0$ for $\mathrm{KV}$ distribution, $U / w_{0}=0.154$ for Gaussian distribution, and $U / w_{0}=0-0.154$ depends on the tune depression for thermal equilibrium distribution.

For this reason, the beam radius ratio $a_{\mathrm{f}} / a_{\mathrm{i}}$ is given by the above Eq. (7) with a tune depression $\sigma / \sigma_{0}$ and with a specified particle distribution. In the general case, Eq. (7) can be numerically solved by a root finding calculation, such as bisection method [17]. 


\section{A. Calculation procedure for arbitrary particle distribution (in general case)}

In an arbitrary particle distribution, the calculation procedure is as follows: $1 . \sigma / \sigma_{0}$ and particle distribution are given. 2. $U / w_{0}$ is obtained at each particle distribution. 3. Using Eq. (9), $h$ is given with $\sigma / \sigma_{0}$ and $U / w_{0}$. 4. From Eqs. (7) and (8), $a_{\mathrm{f}} / a_{\mathrm{i}}$ is calculated with $h$ by using the bisection method. 5. By using Eq. (5), $\varepsilon_{\mathrm{f}} / \varepsilon_{\mathrm{i}}$ is evaluated for $\sigma / \sigma_{0}$ and $a_{\mathrm{f}} / a_{\mathrm{i}}$.

On the other hand, in the case with the constant radius ratio approximation $\left(a_{\mathrm{f}}-a_{\mathrm{i}} \ll a_{\mathrm{i}}\right)$, to replace Eq. (5), the possible emittance growth $\varepsilon_{\mathrm{f}} / \varepsilon_{\mathrm{i}}$ is written as [9]

$$
\frac{\varepsilon_{\mathrm{f}}}{\varepsilon_{\mathrm{i}}}=\left[1+\frac{1}{2}\left(\frac{1}{\sigma^{2} / \sigma_{0}^{2}}-1\right) \frac{U}{w_{0}}\right]^{1 / 2},
$$

instead of the above calculation procedure.

\section{B. Example of calculation procedure for initial Gaussian distribution}

For the initial Gaussian distribution, the calculation procedure is as follows: $1 . \sigma / \sigma_{0}$ is given. 2. $U / w_{0}$ is 0.154 for Gaussian distribution [8,9]. 3. Using Eq. (9), $h$ is given with $\sigma / \sigma_{0}$ and $U / w_{0}$. 4. From Eqs. (7) and (8), $a_{\mathrm{f}} / a_{\mathrm{i}}$ is calculated with $h$ by using the bisection method. 5. By using Eq. (5), $\varepsilon_{\mathrm{f}} / \varepsilon_{\mathrm{i}}$ is evaluated for $\sigma / \sigma_{0}$ and $a_{\mathrm{f}} / a_{\mathrm{i}}$.

On the other hand, according to Eq. (11) (Eq. (14) in Ref. [9]), the possible emittance growth $\varepsilon_{\mathrm{f}} / \varepsilon_{\mathrm{i}}$ is immediately calculated by $\left[1+\left(\sigma_{0}^{2} / \sigma^{2}-1\right) 0.1544 / 2\right]^{1 / 2}$ in the case with the constant radius ratio approximation of $a_{\mathrm{f}}-a_{\mathrm{i}} \ll a_{\mathrm{i}}$ [9].

\section{Example of calculation procedure for thermal equilibrium distribution}

For the thermal equilibrium distribution [8], the calculation procedure is as follows: 1. $\sigma / \sigma_{0}$ is given. 2. $U / w_{0}$ is obtained from $\sigma / \sigma_{0}$ [12,18]. 3. Using Eq. (9), $h$ is given with $\sigma / \sigma_{0}$ and $U / w_{0}$. 4. From Eqs. (7) and (8), $a_{\mathrm{f}} / a_{\mathrm{i}}$ is calculated with $h$ by using the bisection method. 5. By using Eq. (5), $\varepsilon_{\mathrm{f}} / \varepsilon_{\mathrm{i}}$ is evaluated for $\sigma / \sigma_{0}$ and $a_{\mathrm{f}} / a_{\mathrm{i}}$. The number density $n(r)$ of the beam particle in radial direction $r$ with the thermal equilibrium distribution, which is regarded as a preferred equilibrium state of the system, is defined by [8]

$$
\begin{aligned}
n(r) & =\hat{n} \exp \left[-\frac{\gamma_{\mathrm{b}} m \beta_{\mathrm{b}}^{2} c^{2} k_{\beta 0}^{2}}{2 k_{\mathrm{B}} T} r^{2}-\frac{1}{\gamma_{\mathrm{b}}^{2}} \frac{q \phi_{\mathrm{s}}(r)}{k_{\mathrm{B}} T}\right] \\
& =\hat{n} \exp \left[-\frac{q \phi_{\mathrm{tot}}(r)}{k_{\mathrm{B}} T}\right],
\end{aligned}
$$

where $\hat{n}$ is the number density on the axis, $k_{\mathrm{B}} T$ is the beam temperature ( $k_{\mathrm{B}}$ is the Boltzmann constant), $\phi_{\mathrm{S}}(r)$ is the space charge potential, and $\phi_{\text {tot }}(r)$ is the total potential (the sum of the space charge and focusing potentials), respectively.

As implied in Eq. (12), the particle distribution corresponds to the balance between the thermal forces and the total potential (a sum of the external potential and space charge potential) in a thermal equilibrium condition. The tune depression $\sigma / \sigma_{0}$ is related with the profile of the particle distribution as described in Ref. [19]. The profile of the particle distribution gives the nonlinear field energy factor $U / w_{0}$.

The nonlinear field energy factor $U / w_{0}$ in the thermal equilibrium distribution was given as a function of tune depression $\sigma / \sigma_{0}$ [12]. The relation between the nonlinear field energy factor $U / w_{0}$ and the tune depression $\sigma / \sigma_{0}$ was simplified by the fitting formula as $U / w_{0}=a\left(\sigma / \sigma_{0}\right)^{b}$ for $a=0.158205$ and $b=2.04566$ [18].

On the other hand, in the case with the constant radius ratio approximation $\left(a_{\mathrm{f}}-a_{\mathrm{i}} \ll a_{\mathrm{i}}\right)$, the possible emittance growth $\varepsilon_{\mathrm{f}} / \varepsilon_{\mathrm{i}}$ is simply calculated by using Eq. (11) with the nonlinear field energy factor $U / w_{0}$ as the fitting formula and the tune depression $\sigma / \sigma_{0}$ given. As a result, Eq. (11) is replaced by

$$
\frac{\varepsilon_{\mathrm{f}}}{\varepsilon_{\mathrm{i}}}=\left[1+\frac{a}{2}\left(\frac{1}{\sigma^{2} / \sigma_{0}^{2}}-1\right)\left(\frac{\sigma}{\sigma_{0}}\right)^{b}\right]^{1 / 2},
$$

for the thermal equilibrium distribution with the constant radius ratio approximation.

\section{CALCULATION RESUlT OF POSSIBLE EMITTANCE GROWTH DESCRIBED BY FREE-ENERGY PARAMETER WITH AND WITHOUT APPROXIMATION OF CONSTANT RADIUS RATIO}

According to the procedures indicated in the previous section, we calculate the final and initial beam radii ratio, and the possible emittance growth.

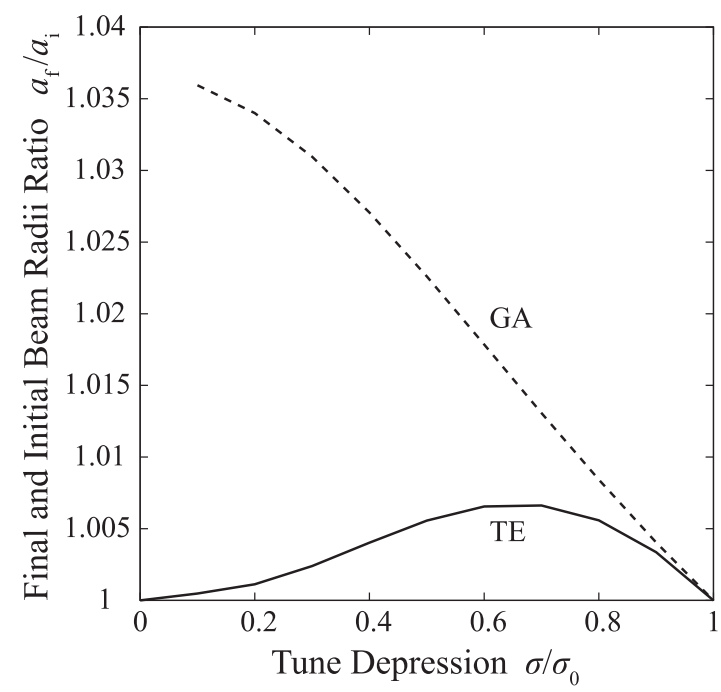

FIG. 1. Final and initial beam radii ratio as a function of tune depression for Gaussian (dashed line) and thermal equilibrium (solid curve) distributions. GA and TE imply Gaussian and thermal equilibrium distributions, respectively. 


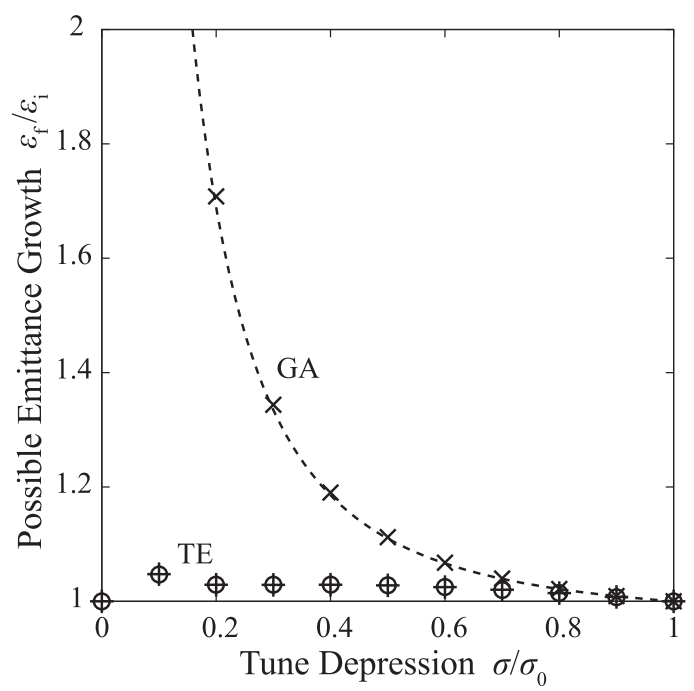

FIG. 2. Possible emittance growth as a function of tune depression and nonlinear field energy factor. The dashed curve and times show the estimation results for Gaussian distribution (GA) with and without constant radius ratio approximation. Circle and plus show the estimation results for thermal equilibrium distribution (TE) with and without constant radius ratio approximation.

Figure 1 shows the final and initial beam radii ratio $a_{\mathrm{f}} / a_{\mathrm{i}}$ as a function of the tune depression $\sigma / \sigma_{0}$ for Gaussian and thermal equilibrium distributions. As shown in Fig. 1, the final and initial beam radii ratio is not exactly constant $\left(a_{\mathrm{f}} / a_{\mathrm{i}} \neq 1\right)$, however it is predicted to be a few percent higher than unity at most. The ratio is expected to be under 1.007 in the whole range of the tune depression for the thermal equilibrium distribution.

Figure 2 shows the possible emittance growth $\varepsilon_{\mathrm{f}} / \varepsilon_{\mathrm{i}}$ as a function of tune depression $\sigma / \sigma_{0}$ and nonlinear field energy factor $U / w_{0}$ with and without the constant radius ratio approximation. An emittance growth of more than a factor of 2 is found at low values of $\sigma / \sigma_{0}$ for the Gaussian distribution, which we attribute to the large nonlinear space-charge forces occurring for this type of distribution. As shown in Fig. 2, the discrepancies are small at each distribution, and it is confirmed that the emittance growths are almost same at each distribution.

\section{CONCLUSION}

In this paper, we developed a procedure to obtain the ratio of the beam radii at the final state and an initial state with arbitrary distribution, and applied it to the estimation of possible emittance growth for Gaussian and thermal equilibrium distributions.

The final and initial beam radii ratio was not exactly constant $\left(a_{\mathrm{f}} / a_{\mathrm{i}} \neq 1\right)$, however the discrepancy was predicted to be a few percent at highest. The ratio was expected to be under 1.007 in the whole range of the tune depression for the thermal equilibrium distribution.

The possible emittance growth as a function of tune depression and nonlinear field energy factor with and without the constant radius ratio approximation was also estimated for both Gaussian and thermal equilibrium distributions. The discrepancies with and without the approximations were small, and it was confirmed that the emittance growths are almost the same at each distribution.

Consequently, to obtain the possible emittance growth, the procedure developed in this paper is more accurate and useful formula in the whole range of the tune depression of beam propagation with arbitrary particle distribution.

[1] K. Horioka, T. Kawamura, M. Nakajima, K. Kondo, M. Ogawa, Y. Oguri, J. Hasegawa, S. Kawata, T. Kikuchi, T. Sasaki, M. Murakami, and K. Takayama, Activities on heavy ion inertial fusion and beam-driven high energy density science in Japan, Nucl. Instrum. Methods Phys. Res., Sect. A 606, 1 (2009).

[2] A. Friedman, J. J. Barnard, R. J. Briggs, R. C. Davidson, M. Dorf, D. P. Grote, E. Henestroza, E. P. Lee, M. A. Leitner, B. G. Logan, A. B. Sefkow, W. M. Sharp, W. L. Waldron, D. R. Welch, and S.S. Yu, Toward a physics design for NDCX-II, an ion accelerator for warm dense matter and HIF target physics studies, Nucl. Instrum. Methods Phys. Res., Sect. A 606, 6 (2009).

[3] R. O. Bangerter, A. Faltens, and P. A. Seidl, Accelerators for inertial fusion energy production, Rev. Accel. Sci. Techol. 06, 85 (2013).

[4] B. Sharkov and D. Varentsov, Experiments on extreme states of matter towards HIF at FAIR, Nucl. Instrum. Methods Phys. Res., Sect. A 733, 238 (2014).

[5] P. A. Seidl, A. Persaud, W. L. Waldron, J. J. Barnard, R. C. Davidson, A. Friedman, E. P. Gilson, W. G. Greenway, D. P. Grote, I. D. Kaganovich, S. M. Lidia, M. Stettler, J. H. Takakuwa, and T. Schenkel, Short intense ion pulses for materials and warm dense matter research, Nucl. Instrum. Methods Phys. Res., Sect. A 800, 98 (2015).

[6] R. C. Davidson and H. Qin, Physics of Intense Charged Particle Beams in High Energy Accelerators (Imperial College Press, Singapore, 2001).

[7] T.P. Wangler, $R F$ Linear Accelerators (Wiley-VCH, New York, 2008).

[8] M. Reiser, Theory and Design of Charged Particle Beams (Wiley, New York, 1994).

[9] M. Reiser, Free energy and emittance growth in nonstationary charged particle beams, J. Appl. Phys. 70, 1919 (1991).

[10] K. Osaki and H. Okamoto, Free-energy formula for emittance-growth estimation in intense mismatched beams, Prog. Theor. Exp. Phys. (2015) 093G01.

[11] J. Struckmeier, J. Klabunde, and M. Reiser, On the stability and emittance growth of different particle phase-space distributions in a long magnetic quadrupole channel, Part. Accel. 15, 47 (1984).

[12] T. Kikuchi and K. Horioka, Static analysis of possible emittance growth of intense charged particle beams with thermal equilibrium distribution, Phys. Plasmas 16, 050703 (2009). 
[13] M. J. L. de Hoon, E. P. Lee, J. J. Barnard, and A. Friedman, Cold phase fluid model of the longitudinal dynamics of space-charge-dominated beams, Phys. Plasmas 10, 855 (2003).

[14] T. Kikuchi, M. Nakajima, K. Horioka, and T. Katayama, Beam instability induced by space charge oscillation during final beam bunching for heavy ion inertial fusion, Phys. Rev. ST Accel. Beams 7, 034201 (2004).

[15] H. Qin, R. C. Davidson, J. J. Barnard, and E. P. Lee, Drift compression and final focus for intense heavy ion beams with nonperiodic, time-dependent lattice, Phys. Rev. ST Accel. Beams 7, 104201 (2004).

[16] P. S. Babu, A. Goswami, and V. Pandit, Transverse dynamics of space charge dominated beam during bunching, Phys. Lett. A 378, 212 (2014).
[17] W. T. V. William H. Press, S. A. Teukolsky, and B. P. Flannery, Numerical Recipes in C: The Art of Scientific Computing (Cambridge University Press, Cambridge, England, 1993).

[18] T. Kikuchi and K. Horioka, Maximum possible emittance growth in static analysis of intense charged particle beams with thermal equilibrium distribution, IEEJ Trans. FM 135, 161 (2015).

[19] S. M. Lund, T. Kikuchi, and R. C. Davidson, Generation of initial kinetic distributions for simulation of longpulse charged particle beams with high space-charge intensity, Phys. Rev. ST Accel. Beams 12, 114801 (2009). 